\title{
Aplicación de la Teoría de Perturbación - Método Diferencial- al Análisis de Sensibilidad en Generadores de Vapor de Centrales Nucleares PWR-Caso Angra I
}

\section{Aplication of the Perturbation Theory- Differential Method- to Sensibility Análisis in PWR Nuclear Power Plant Steam Generator- Angra I}

\author{
Giol Sanders . $^{1}$, Andrade de Lima $F^{2}$., Marques $A^{2}{ }^{2}$, Gallardo $A^{1}$.,Bruna M. ${ }^{1}$, \\ Zúñiga A \\ ${ }^{1}$ Institución Peruano de Energía Nuclear \\ ${ }^{2}$ Universidad Federal de Rio De Janeiro-Brasil
}

\begin{abstract}
RESUMEN
En este trabajo basado en la tesis del Magíster Roberto Giol S. [1] presenta una aplicación del formalismo diferencial de la teoría de perturbación a un modelo termohidráulico homogéneo de simulación del comportamiento estacionario de uno de los generadores de vapor de la Central Nuclear tipo PWR Angra I del Brasil. Se desarrolla un programa de cálculo PERGEVAP tomando como base el código GEVAP de Souza[2]. El programa PERGEVAP permite realizar cálculos de sensibilidad de funcionales lineales (temperatura media del primario)y no lineales (flujo de calor medio a través de las paredes de los tubos del generador) con relación a las variaciones de ciertos parámetros termo-hidráulicos(flujo másico del primario, calor específico, etc), Los resultados obtenidos con este formalismo son luego comparados con los obtenidos del cálculo directo con el propio código GEVAP, pudiéndose verificar una excelente concordancia. Este método se muestra promisorio para efectuar cálculos repetitivos asociados al diseño y análisis de Seguridad de los componentes de las Centrales Nucleares.

Descriptores: teoría de perturbación, método diferencial, sensibilidad, generador de vapor, central nuclear PWR
\end{abstract}

\section{ABSTRACT}

This report presents an application of the differential approach of the perturbation theory to an homogeneous model of a PWR steam generator in the Angra 1 Nuclear Power Plan in Brazil under steady-state conditions. Program PERGEVAP was built fom the code GEVAP developed by Souza and allows sensitivity calculations of linear (average primary loop temperature) and non-linear (average heat flux) functionals due to variations in some thermo-hydraulics parameters (flow rate, specific heat, , etc). Results obtained with this approach are then compared with direct calculations performed using the GEVAP code, with excellent agreements. The method has good potential to treat repeated calculations needed in the design and safety analysis of the Nuclear Plant components.

Keywords: perturbation theory, differential method, steam generator, PWR nuclear Power Plant

\section{INTRODUCCIÓN}

Aplicar la teoría de perturbaciónFormalismo Diferencial- a un modelo homogéneo simplificado que simula el comportamiento estacionario de un generador de vapor de la Central
Nuclear Angra 1 del Brasil, para efectuar un análisis de Sensibilidad de ciertos funcionales relevantes: lineal (temperatura media del refrigerante primario) y no lineal (flujo de calor) con relación a algunos parámetros termo- 
hidráulicos de interés y durante el régimen estacionario de operación de la Central Nuclear.

\section{DESCRIPCIÓN DEL FORMALISMO DIFERENCIAL}

En este método se plantea tres tipos de ecuaciones:

- Ecuaciones directas.

- Ecuaciones Derivadas.

El Problema Directo

Ecuaciones adjuntas.

Considere el problema general de un sistema de $\mathrm{K}$ ecuaciones no-lineales acopladas. Puede escribirse este sistema formalmente como:

$\vec{m} \equiv \vec{m}(\vec{f}, \vec{p}, \vec{r}) \equiv\left[m_{1}, m_{2}, \ldots, m_{k}\right]^{t}=\overrightarrow{0}$

en toda la región del espacio de fase y en donde $\mathrm{m}$ incluye en general operaciones con relación a las variables del espacio de fase.

El vector de las variables de estado, o campo:

$$
\vec{f} \equiv \vec{f}(\vec{p}, \vec{r}) \equiv\left[\mathrm{f}_{1}, \mathrm{f}_{2}, \ldots, \mathrm{f}_{\mathrm{K}}\right]^{\mathrm{t}}
$$

es una función del vector de posición en el espacio de fase:

$$
\vec{r} \equiv\left[\mathrm{r}_{1}, \mathrm{r}_{2}, \ldots, \mathrm{r}_{\mathrm{j}}\right]^{\mathrm{t}}
$$

(Todos los vectores columna son escritos horizontalmente por cuestión de conveniencia solamente)

$Y$ del vector de parámetros de entrada $\vec{p}(\vec{r})$ :

$$
\vec{p} \equiv \vec{p}(\vec{r}) \equiv\left[\mathrm{p}_{1}, \mathrm{p}_{2}, \ldots, \mathrm{p}_{1}\right]^{\mathrm{t}}
$$

Las componentes $\mathrm{mi}(\mathrm{i}=1,2, \ldots \ldots \mathrm{K})$ son funciones no lineales de $f$ y del vector de los parámetros de entrada , $\vec{p}(\vec{r})$ que describen totalmente el sisierna. Este sistema de ecuaciones representa las ecuaciones directas.Las condiciones de contorno del sistema (1) son escritas formalmente como:

$$
\vec{C} \equiv \vec{C}(\vec{f}, \vec{p}, \vec{r})=\overrightarrow{0} \quad\left(\text { en } \vec{r}=\overrightarrow{r_{C}}\right)
$$

donde:

Los vectores $m$ y $C$ incluyen en general las ecuaciones que relacionan a las variables de estado, los parámetros de entrada y las variables independientes contenidas en el vector de posición en el espacio de fase.

\section{El Problema Derivado}

Expandiendo la ecuación perturbada (aquí denotada con el símbolo '), es decir:

$$
\vec{m}^{\prime}\left(\vec{f}^{\prime}, \vec{p}^{\prime}, \vec{r}\right)=\overrightarrow{0}
$$

alrededor de una solución de referencia de $\vec{f}$ ( unívocamente determinada dado que los parámetros $\mathrm{pI}$ son conocidos) hasta el primer orden, se obtiene:

$$
\begin{gathered}
\delta \vec{m}=\vec{m}^{\prime}\left(\vec{f}^{\prime}, \vec{p}^{\prime}, \vec{r}\right)-\vec{m}(\vec{f}, \vec{p}, \vec{r})=\sum_{i=1}^{I} \delta p_{i} \frac{d \vec{m}}{d p_{i}} \\
=\quad \delta p_{i}\left[\frac{\partial \vec{m}}{\partial p_{i}}+H \frac{\partial \vec{f}}{\partial p_{i}}\right]=\overrightarrow{0}
\end{gathered}
$$

Donde $H$ es un operador matricial Jacobiano dado por:

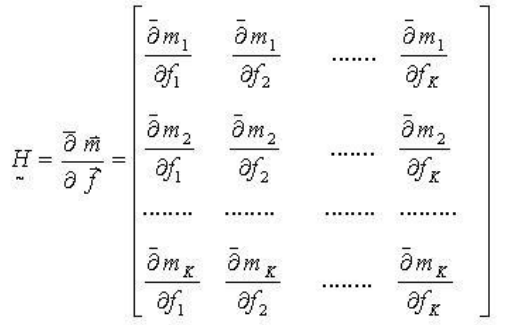

y $\frac{\bar{\partial}}{\partial f_{i}}$ denota una derivada de Frechet

Dado que las perturbaciones $\delta p i$ (admitidas independientes) son arbitrarias, es necesario para que se cumpla la ecuación (7) que:

$$
\left[\frac{\partial \vec{m}}{\partial p_{i}}+H \frac{\partial \vec{f}}{\partial p_{i}}\right]=\overrightarrow{0}
$$

O lo que es lo mismo:

$$
\underset{\sim}{H} \vec{f} / p_{i}=\vec{S}\left(p_{i}\right)
$$

Esta expresión constituye el sistema de ecuaciones derivadas donde:

$$
\underset{\sim}{H}=\frac{\vec{\partial} \vec{m}}{\partial \vec{f}} \quad \vec{f} / p_{i}=\frac{\partial \vec{f}}{\partial p_{i}} \quad \dot{S}\left(p_{i}\right)=-\frac{\partial \vec{m}}{\partial p_{i}}
$$

Las condiciones de borde para las ecuaciones derivadas [ecuación(9)] se obtienen derivando la expresión (5) obteniéndose:

$$
\frac{\partial \vec{C}}{\partial p_{i}}+\frac{\bar{\partial} \vec{C}}{\partial \vec{f}} \cdot \frac{\partial \vec{f}}{\partial p_{i}}=0 \quad\left(\text { en } \vec{r}=\overrightarrow{r_{C}}\right)
$$




\section{ANÁLISIS DE SENSIBILIDAD EL FUNCIONAL DE RESPUESTA}

Considere un funcional respuesta $R$ definido por:

$$
\mathrm{R}=\left\langle\alpha \mathrm{T}_{1}+\beta>\right.
$$

donde:

$\mathrm{S}^{+}$es una función conocida y la integración es realizada en todo el espacio de fase.

Se desea calcular la variación d R resultante de las perturbaciones dpi ( $\mathrm{i}$ $=1,2, \ldots, N)$ de los parámetros del sistema.

El análisis de sensibilidad consiste en evaluar la variación $\mathrm{dR}$ resultante de las perturbaciones dpi $(i=1,2, \ldots, I)$ en algunos o todos los parámetros del sistema..

La variación de $\mathrm{R}$ obtenida a partir de la ecuación (12), está dada por:

$\delta R=\sum_{i=1}^{I} \delta p_{i}\left[\left\langle\vec{f} \cdot \vec{S}^{+} / p_{i}\right\rangle+\left\langle\vec{f} / p_{i} \cdot \vec{S}^{+}\right\rangle\right]$

donde:

$$
\begin{gathered}
\vec{S}^{+} / p_{i}=\frac{\partial \vec{s}^{+}}{\partial p_{i}} \\
\vec{f} / p_{i}=\frac{\partial \vec{f}}{\partial p_{i}}
\end{gathered}
$$

Para obtener $\delta \mathrm{R}$ es necesario evaluar $\vec{f} / p_{i}$

El coeficiente de sensibilidad relativo a un dado parámetro "pi" del sistema resulta:

$$
\left.\frac{\delta R}{\partial p_{i}}=\left[\left\langle\vec{f} \cdot \vec{S}^{+} / p_{i}\right\rangle+<\vec{f} / p_{i} \cdot \vec{S}^{+}\right\rangle\right]
$$

La ecuación (9) junto con la condición de contorno dada por la ecuación (11) permiten evaluar $\vec{f} / \mathrm{p}_{\mathrm{i}}$ valor que sustituido en la ecuavión (16) nos daría el coeficiente de sensibilidad. Sin embargo, a pesar de que la ecuación (9) es lineal dado que el operador matricial y el término fuente no son función del vector de estado derivado ( $\mathrm{H}$ depende de $\vec{f}$ más no de $\vec{f}^{\prime} p_{i}$ ), su solución presenta el inconvenıente de depender del parámetro pi.

Por lo anterior se tiene que por cada parámetro de interés para el estudio de un funcional dado debe resolverse una nueva ecuación como la (9) para obtener el valor $f / p_{i}$ que será utilizado en la ecuación (17). En otras palabras, para cada funcional dado, si se quieren analizar "l" parámetros se tendrán también "l" ecuaciones a resolver.

Es por eso que se recurre al uso de un sistema de ecuaciones adjunto asociado a la ecuación (9) el cual además de permanecer lineal, presenta la ventaja con respecto al anterior sistema de no depender de pi, como se verá a continuación.

\section{Ecuación Adjunta}

Definiendo un operador $\mathrm{H}^{*}$ "Adjunto Extendido" de $\mathrm{H}$, por la expresión:

$\left\langle\vec{f} / p_{i}, H^{*} \cdot \vec{f}^{*}\right\rangle=\left\langle\vec{f}^{*} \cdot \mathrm{H}, \vec{f} / p_{i}\right\rangle+P\left(\vec{f}, \vec{f} / p_{i}\right)$

donde:

$$
\vec{f}_{* \text { : es el vector adjunto de }} \vec{f}_{/ p_{i}}
$$

$\mathbb{P}\left(\vec{f}, \vec{f} / p_{2}\right)$

: es el concomitante bilineal de

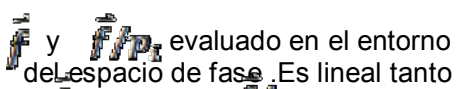
delespacio de fase, Es

Es posible definir el problema adjunto planteando las ecuaciones adjuntas a las ecuaciones (9) y (11) formalmente como sigue:

Ecuación Adjunta: $H^{*} T_{1}{ }^{*}=S^{+}$

Condiciones de contorno adjuntas:

$$
\vec{c}^{\star}\left(\vec{f}^{\star}\right)=\overrightarrow{0}
$$

$\vec{S}^{+}$es el vector de funciones de peso

$\overrightarrow{\mathrm{s}}^{+}=\left[S_{1}{ }^{+}, S_{2}{ }^{+}, \ldots S_{K}{ }^{+}\right]$

Remplazando las ecuaciones (9) y (18) en la ecuación (17) y con las consideraciones anteriores, sta puede ser rescrita como: 


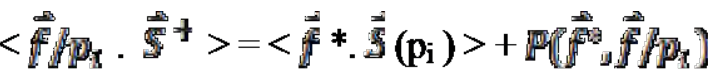

Ecuación que remplazada en la ecuación (13) resulta en:

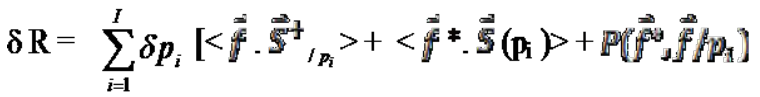

La ecuación (22) permite evaluar $\delta R$ a partir de una solución de la ecuación directa (1) con la condición de contorno dada por la ecuación (2) y una solución de la ecuación adjunta(18), independiente de pi, con la condición de contorno dada por la ecuación(19).

\section{APLICACIÓN AL MODELO HOMOGÉNEO DE GENERADOR DE VAPOR}

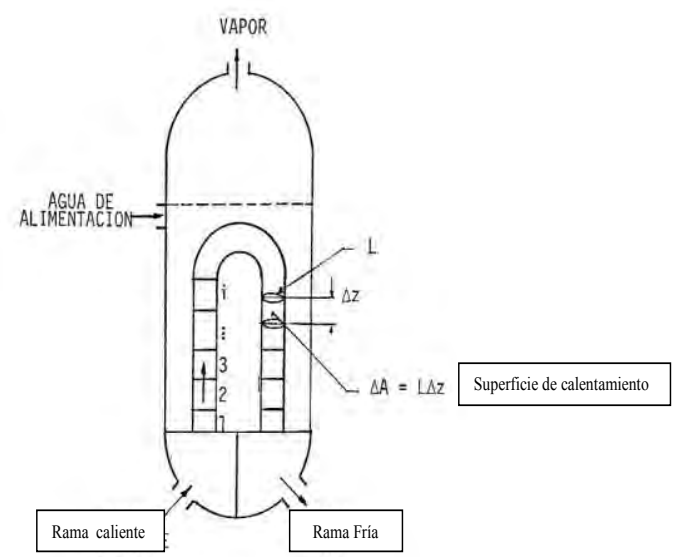

\section{ccuacivies vasicás}

La ecuación directa resulta de aplicar la ecuación de continuidad y la ecuación de conservación de energía para un flujo unidimensional en régimen permanente en el primario. Se demuestra que:

$$
\rho_{1} \cdot V_{1} \frac{d h e_{1}}{d Z}-\frac{d \dot{q}_{1} "}{d Z}=0
$$

donde:

$$
\begin{aligned}
& \rho_{1}: \text { Densidad del fluido primario }\left(\mathrm{Kg} / \mathrm{m}^{3}\right) \\
& V_{l}: \text { Velocidad del fluido primario, }(\mathrm{m} / \mathrm{s}) \\
& h e_{1}: \text { Entalpía específica del fluido primario, }(\mathrm{J} / \mathrm{Kg}) \\
& \dot{q}_{1} \text { ": Flujo de calor a través de la pared del primario, }\left(\mathrm{J} / \mathrm{m}^{2} . \mathrm{s}\right)
\end{aligned}
$$

Asimismo, la ecuación de conducción de calor o Ley de Fourier, aplicada a la pared de transferencia de calor (tubería del circuito primario), resulta en:

$$
\frac{d T_{2}}{d x}-\frac{\left(\tilde{T_{23}}-\tilde{T_{21}}\right)}{d}=0
$$

donde:
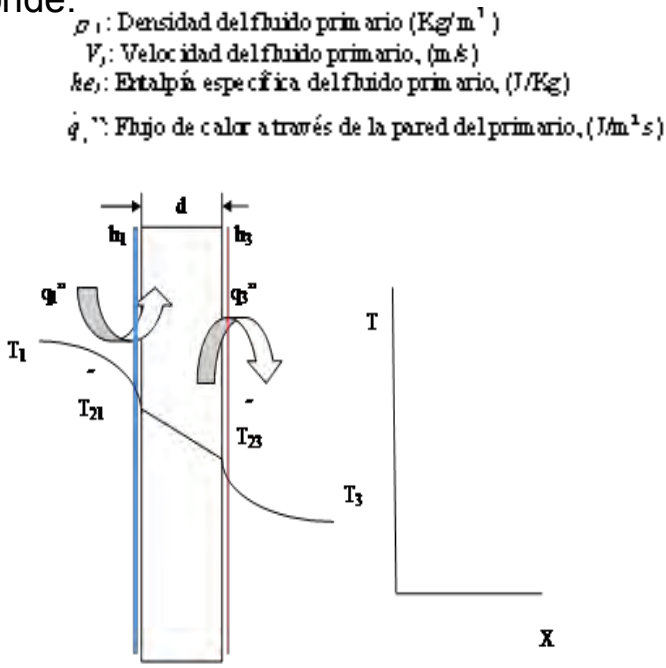

Es posible rescribir la ecuación directa (23) como:

$$
\frac{d T_{1}}{d z}+b\left(T_{1}\right) T_{1}=b\left(T_{1}\right) \cdot T_{S A T}
$$

donde:

$$
\left.\left.\begin{array}{c}
b^{\prime \prime}=b \\
b\left(T_{1}\right)=\frac{h_{1} \cdot A_{T}}{W_{1} \cdot C p_{1} \cdot H_{t}}
\end{array} \frac{\frac{\partial b}{\partial T_{1}}\left(T_{1}\right.}{1+\frac{h_{1}}{h_{3}}+\frac{h_{1} \cdot d}{k_{2}}}\right] T_{S A T}\right)
$$

siendo:

$A_{t}:$ Area total de transferencia de calor, $\left(\mathrm{m}^{2}\right)=$

$$
=N_{t} \cdot \pi \cdot \bar{D} \cdot H_{t}
$$

$N_{t}$ : Número total de tubos del generador de calor $\mathrm{C}_{\mathrm{p} 1}$ : $\quad$ Calor específico del fluido primario, $\left(\mathrm{J} / \mathrm{m}^{2} . \mathrm{s}\right)$

$\bar{D} \quad$ : Diámetro medio de los tubos del generador de calor, (m)

$H_{t}$ : Longitud de cada tubo, $(\mathrm{m})$

$\mathrm{W}_{1}$ : $\quad$ Flujo másico del primario, $(\mathrm{Kg} / \mathrm{s})$

$$
=\rho_{1} \cdot V_{1} \cdot A_{1}
$$

$\mathrm{K}_{2}$ : conductividad térmica de la pared de los tubos $\left(\mathrm{J} / \mathrm{m} . \mathrm{s} .{ }^{\circ} \mathrm{C}\right)$

d : $\quad$ Espesor de la pared de los tubos, (m)

la ec. (23) se puede escribir de otra forma: 
$m\left(T_{1}, p\right)=L T_{1}-\Phi=0$

donde:

$$
\begin{gathered}
\underset{\sim}{L}=\frac{d}{d z}+b\left(T_{1}\right) \\
\Phi=\mathrm{b}_{\mathrm{SAT}}
\end{gathered}
$$

(Por comodidad llamaremos $a b(T 1)=b$ de aquí en adelante.)

Luego, debido pues a las hipótesis simplificadoras adoptadas, el problema se reduce al cálculo del campo de temperaturas $\mathrm{T} 1$, obteniéndose las demás temperaturas (T2 y $\mathrm{T} 3$ ) en función de T1.

Derivando la ec.(26) con relación a un parámetro "p" cualquiera :

$$
\frac{d m}{d p}=\frac{\partial m}{\partial T_{1}} \frac{\partial T_{1}}{\partial p}+\frac{\partial m}{\partial p}=0
$$

se obtiene la ecuación derivada que puede ser escrita como:

$$
\mathrm{H} \mathrm{T}_{1} / \mathrm{p}=\mathrm{S}(\mathrm{p})
$$

donde se demuestra que:

$$
\begin{gathered}
H=\frac{\partial \bar{m}}{\partial T_{1}}=\frac{\partial}{\partial z}(.)+b^{\prime \prime}(.) \\
b^{\prime \prime}=b+\frac{\partial b}{\partial T_{1}}\left(T_{1}-T_{S A T}\right) \\
\mathrm{T}_{1} / \mathrm{p}=\frac{\partial \mathrm{T}_{1}}{\partial \mathrm{p}} \\
\vec{S}\left(\mathrm{p}_{\mathrm{i}}\right)=-\frac{\partial m}{\partial p_{i}}=-\frac{\partial b}{\partial p_{i}}\left(T_{1}-T_{S A T}\right)+b \frac{\partial T_{S A T}}{\partial p_{i}}
\end{gathered}
$$

\section{El Funcional de Respuesta}

Consideremos un funcional o respuesta genérica no lineal

$$
R=\left\langle\alpha T_{1}+\beta>\right.
$$

Donde:

$\alpha=f_{1}\left(T_{1}, p_{i}\right)$

$\beta=f_{2}\left(T_{1}, p_{i}\right)$

<.(.) > Representa la integración efectuada a todo lo largo de los tubos del generador de vapor l coeficiente de sensibilidad de "R", con relación al parámetro "pi", es obtenido derivando la ecuación (28) con relación a "pi", esto es:

$$
\frac{d R}{d p_{i}}=<S^{+} T_{1 / p_{i}}>+<\frac{\partial \alpha}{\partial p_{i}} T_{1}>+<\frac{\partial \beta}{\partial p_{i}}>
$$

$$
S^{+}=\left\lfloor\alpha+\frac{\partial \alpha}{\partial T_{1}} T_{1}+\frac{\partial \beta}{\partial T_{1}}\right\rfloor
$$

\section{Caso Particular}

Un caso particular que se presenta con frecuencia es aquel en el cual "R" es lineal con respecto a T1. En este caso, $\alpha$ es independiente de $T_{1}$ y $\beta=0$ y las ecuaciones (29) y (30) se transforman en:

$\frac{d R}{d P_{i}}=<S^{+} T_{1 / p_{i}}>+<S^{+} / p_{i} T_{1}>$

$S^{+}=\alpha$

El coeficiente de sensibilidad $\mathrm{dR} / \mathrm{dpi}$ puede calcularse a partir de la ecuación (31) siempre y cuando se conozcan $\mathrm{T} 1$ y $\mathrm{T}_{1} / \mathrm{p}_{\mathrm{i}}$.

Las ecuaciones (26) y (27) permiten obtener el valor de $\mathrm{T}_{1}$ y $\mathrm{T}_{1} / \mathrm{p}_{\mathrm{i}}$ respectivamente. Sin embargo, la solución de la ecuación (27) presenta el inconveniente de depender del parámetro "pi". Este problema puede ser resuelto y evitado hallando la ecuación adjunta de la ecuación (27).

\section{El Problema Adjunto y el Concomitante Bilineal}

De la definición de operador adjunto extendildo, anteriormente mencionado, se tiene que:

$<T_{1 / p_{i}} \underset{\sim}{H} T_{1}^{*} T_{1}^{*}>=<T_{1}^{*} \underset{\sim}{H} T_{1 / p_{i}}>+P\left(T_{1}^{*}, T_{1 / p_{i}}\right)$

Escribiendo el sistema adjunto como:

donde:

$$
H^{*} T_{1}^{*}=S^{+}
$$


$\mathrm{S}^{+}=$Término de fuente relacionado con el funcional $R$, a través de las ecuaciones (28) y (30).

Se demuestra que:

$$
H_{\sim}^{*}=-\frac{\partial}{\partial z}+b^{\prime \prime}
$$

donde:

$$
b^{\prime \prime}=b+\frac{\partial b}{\partial T_{1}}\left(T_{1}-T_{S A T}\right)
$$

siendo:

y además:

$$
b=b\left(T_{1}\right)=\frac{h_{1} A_{T}}{W_{1} C p_{1} H_{t}}\left[\frac{1}{1+\frac{h_{1}}{h_{3}}+\frac{h_{1} d}{k_{2}}}\right]
$$

$$
\begin{aligned}
& P\left(T_{1}^{*}, T_{1 / p_{1}}\right)=T_{10} * \frac{\partial}{\partial p} T_{11} \\
& =T_{10} * T_{10 / \gamma_{1}}
\end{aligned}
$$

donde

$$
\begin{aligned}
T_{10} & =T_{1}(z=0) \\
T_{10} * & =T_{1} *(z=0)
\end{aligned}
$$

Reemplazando estos valores en la ecuación (34) se obtiene la ecuación adjunta de la ecuación (27):

$$
-\frac{\partial T_{1} *}{\partial z}+b^{\prime \prime} T_{1} *=S^{+}
$$

Esta ecuación es lineal, dado que b" depende de $\mathrm{T} 1$ más no de $\mathrm{T} 1{ }^{*}$ y su solución es independiente de las variaciones del parámetro "pi".

La condición de contorno, impuesta por conveniencia, es:

$$
T_{1}^{*}\left(z=H_{t}\right)=0
$$

o sea, el valor de la función importancia es nulo en el punto final.

Sustituyendo las ecuaciones (27), (34) y (37) en la ecuación (33) se obtiene:

$<T_{1 / p_{i}} S^{+}>=<T_{1} * S\left(p_{i}\right)>+T_{10} * T_{10} / p_{i}$

Finalmente, sustituyendo (39) en (29) se obtiene la expresión del coeficiente de sensibilidad de " $R$ " con relación al parámetro arbitrario " $p$ ", esto es:

$\left.\frac{d R}{d p_{i}}=\left\langle T_{1} * S\left(p_{i}\right)\right\rangle+T_{10} * T_{10 / p_{i}}+<\alpha_{\mid p_{i}} \cdot T_{1}>+<\left.\beta\right|_{p_{i}}\right\rangle$

De este modo, resolviendo las ecuaciones (25) y (38) podemos pues calcular $\mathrm{dR} / \mathrm{dpi}$ a partir de la ecuación (40) ya que $a, b$ y y $S+$ son conocidos con la definición de $\mathrm{R}$ y piy el valor de S(pi) es calculado (ver ec. 27).

Discretizando la ecuación (40) esta puede ser escrita aproximadamente :

$$
\left.\Delta R=\sum_{i=1}^{N}\left\{T_{1_{j}} * S\left(p_{i}\right)+T_{10} * T_{10 / p_{i}}+\alpha_{/ p_{i}} T_{1_{i}}+\beta_{/ p_{i}}\right] \Delta p_{i}\right\} \Delta z
$$

\section{DESCRIPCION DEL CASO ANALIZADO}

FUNCIONAL: TEMPERATURA MEDIA DEL FLUIDO PRIMARIO ( $\left.\bar{T}_{1}\right)$

En este caso:

$$
\bar{T}_{1}=\frac{1}{H_{t}} \int_{0}^{H_{t}} T_{1}(z) d z
$$

De la ecuación (29), por definición tenemos:

$$
R_{1}=\bar{T}_{1}=\int_{0}^{H_{t}}\left(\alpha_{1} T_{1}+\beta_{1}\right) d z
$$

Comparando (42) con (43) se deduce que:

$$
\begin{aligned}
& \alpha_{1}=\frac{1}{H_{t}} \\
& \beta_{1}=0
\end{aligned}
$$

y de la ecuación (30) se obtiene:

$$
S^{+}=\frac{1}{H_{t}}
$$

En este caso, para el análisis de sensibilidad fueron seleccionados los siguientes parámetros:

Cp1, h1, h3, K2, W1. 


\section{RESULTADOS Y DISCUSION}

La Tabla 1 muestra los resultados de los cálculos de sensibilidad de la temperatura media del fluido primario con relación a uno de los parámetros seleccionados.(Cp1)

En dichas Tablas, RNP representa la respuesta no perturbada. RP.DIF la respuesta perturbada calculada a partir del formalismo diferencial y RPDIR, la respuesta perturbada calculada directamente a través del modelo de simulación. " $\Delta$ " representa el módulo de la diferencia relativa entre RP.DIF y RP.DIR y finalmente, representa la sensibilidad del funcional "R" relativa al parámetro "pi" y es definida como:

$$
R_{/ p_{i}}=\frac{(R P . D I F-R N P) / R N P}{\delta p_{i} / p_{i}}
$$

Evidentemente, una vez calculado $R_{t p}$ para algún valor cualquiera de dpi /pi, se podrán calcular los demás valores de RP.DIF, para cada valor de dpi /pi de los parámetros analizados, directamente utilizando la ecuación (46).

Tabla 1: Resultados de sensibilidad de la temperatura media del fluido primario con relación al calor específico del primario.(Cp1)

\begin{tabular}{|c|c|c|c|c|c|c|c|}
\hline$\overline{\mathrm{R}}$ & $\begin{array}{l}\text { RNP } \\
\left({ }^{\circ} \mathrm{C}\right)\end{array}$ & $\mathrm{p}$ & $\begin{array}{c}\delta p / p, \\
\%\end{array}$ & $\begin{array}{r}\text { RP.DIF } \\
\left({ }^{\circ}\right)\end{array}$ & $\begin{array}{c}\text { RP.DIR } \\
\left({ }^{\circ} \mathrm{C}\right)\end{array}$ & $\begin{array}{l}\Delta \\
(\%)\end{array}$ & $\begin{array}{l}R_{/ p_{p}} \\
(x 10)\end{array}$ \\
\hline \multirow{10}{*}{$\bar{T}_{1}$} & \multirow{10}{*}{301.540} & \multirow{10}{*}{$C_{p 1}$} & 5 & 302.526 & 302.507 & 0.01 & \multirow{10}{*}{0.65398} \\
\hline & & & 4 & 302.329 & 302.327 & 0.00 & \\
\hline & & & 3 & 302.131 & 302.144 & 0.00 & \\
\hline & & & 2 & 301.934 & 301.960 & -0.01 & \\
\hline & & & 1 & 301.737 & 301.773 & -0.01 & \\
\hline & & & -1 & 301.342 & 301.393 & -0.02 & \\
\hline & & & -2 & 301.145 & 301.200 & -0.02 & \\
\hline & & & -3 & 300.948 & 301.004 & -0.02 & \\
\hline & & & -4 & 300.751 & 300.807 & -0.02 & \\
\hline & & & -5 & 300.553 & 300.607 & -0.02 & \\
\hline
\end{tabular}

\section{GRAFIC0 1}

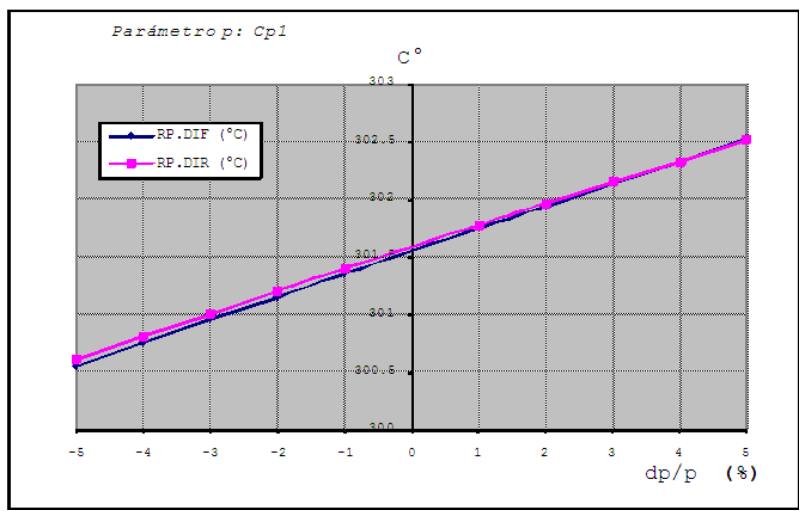

OBSERVACIONESY CONCLUSIONES

Como conclusión podemos afirmar que el programa desarrollado para la aplicación de la Teoría de Perturbación-Formalismo Diferencialen el análisis de sensibilidad en generadores de vapor del tipo de tubos en $U$ de Centrales Nucleares PWR, como el de la central Angra 1 del Brasil - resulta adecuado y permite analizar diferentes parámetros térmicos.

A diferencia de un análisis típico de sensibilidad usando las ecuaciones directas, que requiere muchas corridas $y$ deben realizarse para cada parámetro y para cada orden de magnitud o variación de los parámetros, las ecuaciones del formalismo perturbativo diferencial son lineales, de solución rápida permitiendo obtener valores de sensibilidad para diversos parámetros termo-hidráulicos con un solo cálculo inicial (una sola corrida del programa desarrollado).

\section{AGRADECIMIENTOS}

A los Drs. Carlos Marques Alvim y Fernando Andrade Lima de la UNFRJRio de Janeiro por su asesoría en Brasil y a los Srs. Magíster Alberto Gallardo Padilla, asesor en el IPENLima-Perú, y Agustín Zúñiga y Rubén Bruna por su contribución al desarrollo de este trabajo. 


\section{REFERENCIAS}

[1] GIOL S.R.,A, "Aplicación de la Teoría de Perturbación Formalismo Diferencial- para el análisis de sensibilidades de variables termo-hidráulicas de un generador de vapor de la Central Nuclear Angra 1 del Brasil", Tesis para la obtención del Grado de Magíster en Energía Nuclear en la Universidad Nacional de Ingeniería-Facultad de Ciencias, Lima, Diciembre del 2010.
[2] LOBO DE SOUZA, A., "Modelo Homogéneo de um Gerador de Vapor para

Simulacao de transitorios Operacionais e accidentes em Centráis Nucleares tipo

PWR". Tesis de Maestría IME (1981). 